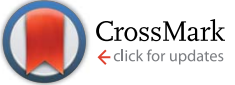

Cite this: RSC Adv., 2015, 5, 89577

\title{
Synthesis, optical properties, and photochemical activity of zinc-indium-sulfide nanoplates $\uparrow$
}

\begin{abstract}
Björn Kempken, ${ }^{a}$ Volodymyr Dzhagan, ${ }^{\text {bc }}$ Dietrich R. T. Zahn, ${ }^{\text {b }}$ Marcelo J. P. Alcocer, ${ }^{d}$

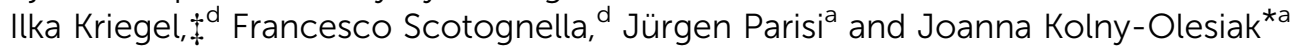

Zinc indium sulfide is an interesting material for photocatalytical applications. Here, $Z n_{x} \mid n_{2} S_{3+x}$ nanoplates with varying value of $x$ (between 0 and 1 ) were synthesized by means of colloidal chemistry. The nanoplates have a diameter of up to $100 \mathrm{~nm}$ and a relatively small thickness of $2.5 \mathrm{~nm}$. The structure of the $\mathrm{Zn}$-free particles could be assigned to $\beta-\ln _{2} S_{3}$ based on the powder $X$-ray diffraction and Raman spectroscopy data. Particles containing $\mathrm{Zn}$ exhibit the hexagonal $\mathrm{ZnIn}_{2} \mathrm{~S}_{4}$ structure. The samples were studied by steady state and time resolved photoluminescence spectroscopy. Our results demonstrate that increasing amounts of $\mathrm{Zn}$ incorporation result in more intra-gap states that participate in radiative subband gap recombination and simultaneously decrease non-radiative defect states that might, for example, originate from vacancies in the structure. Furthermore, the nanoplates exhibit significantly increased photochemical activity in the degradation of rhodamine 6G, depending on their Zn content. The high surface-to-volume ratio and the intra-gap states might be responsible for this finding.
\end{abstract}

Received 1st August 2015 Accepted 9th October 2015

DOI: $10.1039 / \mathrm{c} 5 \mathrm{ra} 20570 \mathrm{k}$

www.rsc.org/advances interesting thermoelectrical properties, ${ }^{14}$ while the hexagonal modification exhibits photoluminescence and photoconductivity. Both polymorphs are photocatalytically active materials. ${ }^{13,15-20}$ Nanoribbons, nanotubes, and microspheres of hexagonal $\mathrm{ZnIn}_{2} \mathrm{~S}_{4}$ have been synthesized in a solvothermal reaction, ${ }^{21}$ while nanowires could be obtained by using a porous polycarbonate membrane as hard template..$^{20}$ Nanoparticles with a size between 2.1 and $9.2 \mathrm{~nm}$ and large, aggregated nanoplates were obtained by means of colloidal chemistry. ${ }^{19}$ Also other zinc-indium-sulfide microspheres with the general stoichiometry $\mathrm{Zn}_{x} \mathrm{In}_{2} \mathrm{~S}_{3+x}(x \geq 1)$ were synthesized by a solvothermal method, and their photocatalytic activity in water splitting reaction has been tested. ${ }^{22}$ However, there are no reports about the formation of well-defined $\mathrm{Zn}_{x} \operatorname{In}_{2} \mathrm{~S}_{3+x}$ nanoplates.

In this article, we present the colloidal synthesis of ultrathin, hexagonal $\mathrm{Zn}_{x} \mathrm{In}_{2} \mathrm{~S}_{3+x}$ nanoplates (with $0 \leq x \leq 1$ ) and show their capability to act as photocatalysts for the degradation of organic dyes. The nanoplates were synthesized using different amounts of zinc, and their composition dependent structure, optical properties, and photocatalytic activity were studied.

\section{Experimental section}

\section{Materials}

Indium(III)acetate $\left(99.99 \%-\right.$ In) $\left(\mathrm{InAc}_{3}\right)$, copper(I)acetate $(99.99 \%-\mathrm{Cu})(\mathrm{CuAc})$, zinc(II)acetate $(99.99 \%-\mathrm{Zn})\left(\mathrm{ZnAc}_{2}\right)$, oleylamine (OLAM), oleic acid (90\%, technical grade) (OA), 1dodecanethiol (1-DDT), and tert-dodecanethiol $(t$-DDT) were 
purchased from Sigma Aldrich. All the chemicals were used without further purification.

\section{Preparation of Cu-stock solution}

$1 \mathrm{mmol} \mathrm{CuAc}$ was dissolved in $10 \mathrm{~mL}$ OLAM and heated under stirring and inert gas up to $100^{\circ} \mathrm{C}$ for $10 \mathrm{~min}$. The solution was stored under argon.

\section{Synthesis of the nanoplates}

In a typical synthesis, $1 \mathrm{mmol} \operatorname{InAc}_{3}, 100 \mu \mathrm{L} \mathrm{Cu}$-stock solution, 1 $\mathrm{mmol} \mathrm{ZnAc}_{2}, 10 \mathrm{~mL}$ OLAM, and $2 \mathrm{~mL} \mathrm{OA}$ were loaded in a threeneck-flask and stirred under vacuum at room temperature for $15 \mathrm{~min}$. Subsequently, the reaction solution was heated up to $280{ }^{\circ} \mathrm{C}$ under nitrogen atmosphere. At this temperature, a mixture of $0.25 \mathrm{~mL} \mathrm{1-DDT}$ and $2.5 \mathrm{~mL} t$-DDT was rapidly injected. After additional $60 \mathrm{~min}$, the solution was cooled down to room temperature, and the resulting nanoplates were isolated from the by-products of the reaction and the remaining starting materials by precipitation with ethanol and subsequent redissolution in hexane; the latter was repeated up to five times. Afterwards, the purified nanoplates were dissolved in chlorobenzene.

\section{Degradation of rhodamine 6G}

$2 \mathrm{~mL}$ rhodamine $6 \mathrm{G}$ solution $\left(100 \mu \mathrm{mol} \mathrm{L}^{-1}\right)$ was added to $1 \mathrm{~mL}$ nanoplates solution $\left(1 \mathrm{mg} \mathrm{mL}^{-1}\right)$. The resulting solution was illuminated with UV light ( $365 \mathrm{~nm}$ ) for a total time of $10 \mathrm{~min}$. Absorption spectra were taken at different irradiation intervals (between $10 \mathrm{~s}$ and $10 \mathrm{~min}$ ), in order to monitor the decomposition of the dye.

\section{Characterization}

Transmission electron microscopy (TEM) images were taken on a Zeiss EM902A electron microscope using an $80 \mathrm{kV}$ electron source. Samples were prepared from a hexane solution on a carbon coated copper grid and dried under air for 24 hours. High resolution transmission electron microscopy (HRTEM) observations as well as energy dispersive X-ray spectroscopy (EDX) measurements were performed on a JEOL JEM2100F electron microscope equipped with an X-Max80 SDD-detector. UV/vis-absorption spectra were taken on a Varian Carry 100 scan spectrophotometer, and samples were prepared by diluting a 5-50 $\mu \mathrm{L}$ nanocrystal solution with $3 \mathrm{~mL} n$-hexane. PL spectra were taken on a Fluorolog 3. Powder X-ray diffraction (XRD) was measured with a PANalytical X'Pert PRO MPD diffractometer using $\mathrm{Cu} \mathrm{K} \alpha$ radiation (1.54 $\mathrm{\AA}$ ), standard BraggBrentano $\theta-2 \theta$ geometry, and variable slits. The samples were measured on low background silicon sample holders and prepared by dropping colloidal solution on the holder and letting the solvent evaporate (by heating to $70{ }^{\circ} \mathrm{C}$ for $30 \mathrm{~min}$ ). Raman spectra were excited with the $514.7 \mathrm{~nm}$ line of diode pumped solid state (DPSS) laser (Cobolt) or a $325 \mathrm{~nm}$ line HeCd laser and registered with an HR LabRam spectrometer, equipped with a liquid nitrogen cooled charge-coupled device (CCD) detector, at a spectral resolution of $5 \mathrm{~cm}^{-1}$. The incident laser power was kept below $0.02 \mathrm{~mW}$, in order to avoid sample heating under the microscope objective $(100 \times$ and $40 \times$ for $514.7 \mathrm{~nm}$ and $325 \mathrm{~nm}$ excitation, respectively). Time-resolved photoluminescence measurements were performed using a femtosecond laser source and streak camera detection system. An unamplified Ti:sapphire laser (Coherent Chameleon Ultra II) operating at $80 \mathrm{MHz}$ was tuned to provide pulses with central wavelengths of $800 \mathrm{~nm}$, energies of $\sim 50 \mathrm{~nJ}$, and temporal and spectral bandwidths of $\sim 140$ fs and $\sim 5 \mathrm{~nm}$, respectively. An acousto-optical modulating pulse picker (APE Pulse Select) was used to reduce the repetition rate to $2 \mathrm{MHz}$ to accommodate the long decay times of the samples. A $\beta$-barium borate crystal provided type I phase-matched second harmonic generation, leading to pulses with central wavelengths of $400 \mathrm{~nm}$. These were focused onto the sample, maintaining a low fluence $(<30$ $\mathrm{mJ} \mathrm{cm}{ }^{-2}, \sim 100 \mu \mathrm{m}$ spot diameter) in order to avoid any saturation effects in the sample. The resulting collected emission was analyzed by a spectrograph (Princeton Instruments Acton SP2300) coupled to a streak camera (Hamamatsu C5680) equipped with a linear voltage sweep module. In this way, measurements of photoluminescence intensity as a function of both wavelength and time were obtained with spectral and temporal resolutions of $\sim 1 \mathrm{~nm}$ and $\sim 0.5 \mathrm{~ns}$ respectively. Temporal broadening of the pump pulses caused by dispersive elements was confirmed to be well below the response time of the detection system.

\section{Results and discussion}

The photocatalytic activity of nanostructured materials depends on their electronic properties and the characteristics of their surface. This study concentrates on the synthesis and characterization of nanocrystalline $\mathrm{Zn}_{x} \mathrm{In}_{2} \mathrm{~S}_{3+x}$ with high specific surface area and variable composition. The manuscript is organized in the following manner: in the first part we describe the synthesis of the $\mathrm{Zn}_{x} \mathrm{In}_{2} \mathrm{~S}_{3+x}$ nanoplates, and then we present their detailed structural and spectroscopic characterization. We conclude with the demonstration of the photochemical activity of the synthesized nanoplates in the decomposition of rhodamine G6.

\section{Colloidal synthesis of zinc indium sulfide nanoplates}

The starting materials for the formation of $\mathrm{Zn}_{x} \mathrm{In}_{2} \mathrm{~S}_{3+x}$ nanoplates are zinc and indium acetate. The source of sulfur is tertdodecanethiol, a compound that can be easily decomposed thermally and provides the reaction solution with sulfur monomers. Therefore, it has been used as a sulfur source in the synthesis of metal sulfides, such as CuInS $2, \mathrm{Cu}_{2-x} \mathrm{~S}$, or $\mathrm{Cu}_{3} \mathrm{SnS}_{4}$ nanoparticles (NPs). ${ }^{23-27}$ 1-Dodecanethiol and oleic acid act as stabilizers, which not only provide the emerging particles with colloidal stability, but also regulate the activity of the monomers in the reaction solution. The reaction takes place in oleylamine, which is a coordinating solvent and can also play a role of a stabilizer for the growing particles. A small amount of copper acetate (indium/copper ratio $100: 1$ ) is present in the reaction solution, because it facilitates the formation of the nanoplates. 
The composition of the nanoplates depends on the amount of zinc used for the synthesis. When comparing the ratio of the starting materials to that of the elements comprising the nanoplates, only about $50 \%$ of the expected amount of zinc is incorporated into the nanoplates. The composition of the different samples, obtained by energy dispersive X-ray spectroscopy (EDX) measurements can be found in Table 1. By changing the amount of zinc in the reaction solution, we can tune the composition of the $\mathrm{Zn}_{x} \mathrm{In}_{2} \mathrm{~S}_{3+x}$ nanoplates between $x=$ 0 and $x=1$. However, compared to the anticipated $Z_{x} \operatorname{In}_{2} S_{3+x}$ stoichiometry, the sulfur content is larger than expected for all samples. This is most likely due to the presence of thiols on the surface of the particles. It has to be noted that we also detect a small amount of copper in all measurements; however, it is not possible to determine the exact concentration of copper ions in the samples, as this element is always also present as a background signal. Due to the small concentration of copper ions in the samples, subtraction of the background values does not give reliable results. Because the concentration of copper in all reaction solutions is the same, we can safely assume that it also does not vary significantly among all the samples studied, and we can attribute the differences observed between them to the variation of the $\mathrm{Zn}$ concentration.

\section{Structural characterization of zinc indium sulfide nanoplates}

Fig. 1 shows transmission electron microscopy (TEM) images of the nanocrystals obtained by our synthetic procedure. The particles are thin nanoplates with a diameter up to $100 \mathrm{~nm}$ independent of the amount of zinc used for the synthesis. Many of them have regular, hexagonal or triangular shapes with $120^{\circ}$ or $60^{\circ}$ angles, respectively (Fig. $1 \mathrm{~b}$ and S1 of the ESI $\dagger$ ). Others have irregular shapes, with torn away edges, which we attribute to their low mechanical stability during the vigorous stirring process and the precipitation, centrifugation and redissolution steps during the cleaning procedure (Fig. 1a). Most nanoplates are located flat on the TEM grid, however, in some larger aggregates, bundles of several plates lying on the edge could be found (Fig. 1c-e), allowing their thickness to be determined at $2.5 \mathrm{~nm}$. This is close to the value of the $c$-axis of hexagonal $\mathrm{ZnIn}_{2} \mathrm{~S}_{4}$ (ICDD 03-065-2023), which is $2.4 \mathrm{~nm}$. This crystallographic structure is composed of a repeating unit of 4 layers of densely packed sulfur ions of $1.2 \mathrm{~nm}$ thickness with one layer of

Table 1 Composition of samples prepared with different $\mathrm{Zn}$ content of the reaction solution measured by EDX

\begin{tabular}{llllll}
\hline & $\begin{array}{l}\mathrm{ZnAc}_{2} \text { in the } \\
\text { reaction solution }[\mathrm{g}]\end{array}$ & $\begin{array}{l}\text { Zn/In ratio in } \\
\text { the reaction solution }\end{array}$ & $x \mathrm{Zn}$ & $\operatorname{In}^{a}$ & $(3+x) \mathrm{S}$ \\
\hline ZIS250 & $0.250 \mathrm{~g}$ & $1.37: 1$ & 1.05 & 2 & 5.94 \\
ZIS150 & 0.150 & $0.85: 1$ & 0.79 & 2 & 5.10 \\
ZIS100 & $0.100 \mathrm{~g}$ & $0.57: 1$ & 0.61 & 2 & 4.55 \\
ZIS50 & $0.050 \mathrm{~g}$ & $0.27: 1$ & 0.30 & 2 & 4.31 \\
ZIS25 & $0.025 \mathrm{~g}$ & $0.14: 1$ & 0.18 & 2 & 3.82 \\
ZIS13 & $0.0125 \mathrm{~g}$ & $0.07: 1$ & 0.09 & 2 & 3.71 \\
ZIS0 & $0.00 \mathrm{~g}$ & $0.00: 1$ & 0 & 2 & 3.49
\end{tabular}

${ }^{a}$ The value for In was normalized to 2 .
$\mathrm{Zn}$ ions in the tetrahedral voids, one layer of In ions in octahedral voids, and one layer of In ions in tetrahedral voids. This should result in a relatively low contrast in high resolution transmission electron microscopy (HRTEM) images of single nanoplates lying flat on the substrate and in a strong broadening of powder X-ray diffraction (XRD) reflections corresponding to $(00 l)$ lattice planes.

Fig. 2 shows the XRD patterns of the products for different Zn contents. The position of the reflections and their relative intensities are independent of the particle's composition. A similar behavior was reported previously for $\mathrm{Zn}_{x} \mathrm{In}_{2} \mathrm{~S}_{3+x}$ particles, which showed comparable profiles of their diffraction patterns in spite of their differing compositions ( $x=1$ to 5$)^{22}$

Notably, in our case, the observed intensities of the measured reflections substantially differ from those of the typical reference patterns (ICDD 01-084-1385 for $\beta$ - $\operatorname{In}_{2} \mathrm{~S}_{3}$, Fig. 2, top panel, green pattern and ICDD 03-065-2023 for $\mathrm{ZnIn}_{2} \mathrm{~S}_{4}$, bottom panel, black pattern). This deviation from the intensity values of the bulk reference patterns and the pronounced broadening of some reflections result from the anisotropic growth of the particles. In order to compare the reference patterns with the measured data, we have to take into account the shape of the nanocrystals.

As a rough estimate of the changes expected in the intensity (bulk $v s$. thin nanoplate), we calculated the number of lattice planes present in a nanoplate of $2.4 \mathrm{~nm}$ thickness and a diameter of $30 \mathrm{~nm}$ for each reflection of the reference pattern (black and green sticks in Fig. 2). We assumed a growth perpendicular to the $\langle 001\rangle$ and $\langle 111\rangle$ direction for the hexagonal $\mathrm{ZnIn}_{2} \mathrm{~S}_{4}$ and for the cubic $\beta$ - $\operatorname{In}_{2} \mathrm{~S}_{3}$, respectively. The numbers obtained from this calculation were used to weigh the intensity values of the reference patterns. The resulting values are shown as red and

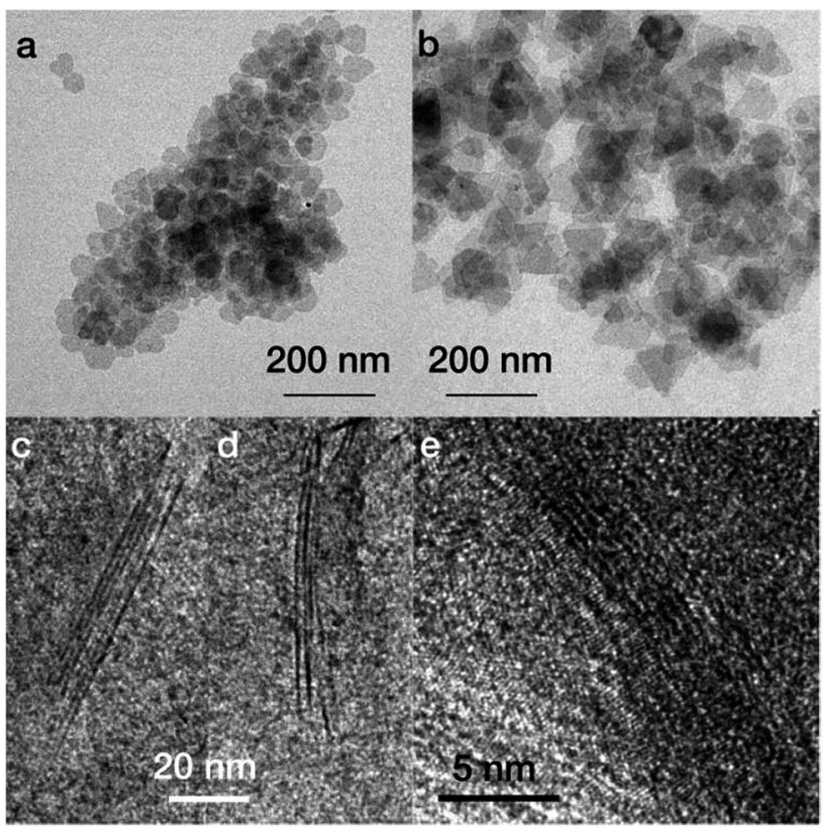

Fig. 1 Overview TEM images of the nanoplates after five precipitation and dissolution steps (a) and without any cleaning procedure (b). TEM images of nanoplates lying on the edge $(c-e)$. 


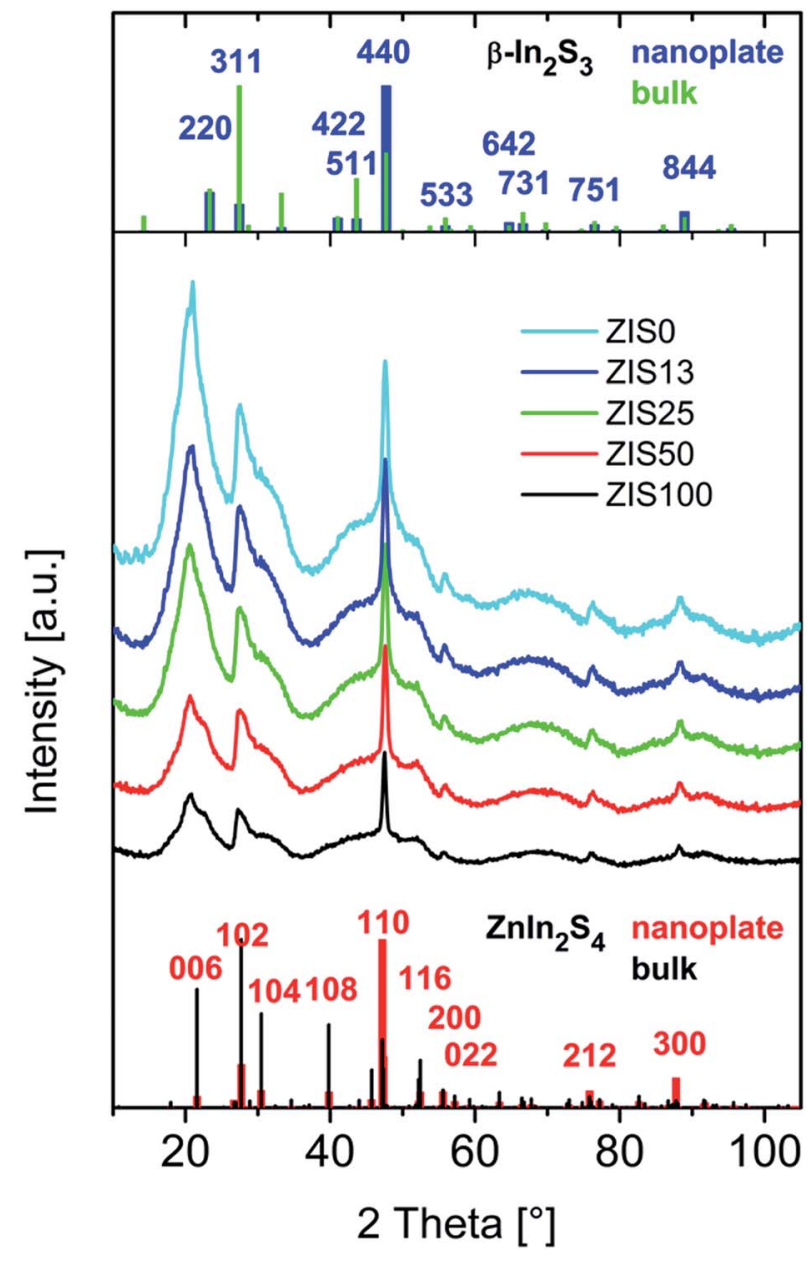

Fig. 2 XRD patterns of nanoplates obtained with different $Z n$ amounts in the reaction solution. The reference patterns are ICDD 01-084-1385 for $\mathrm{In}_{2} \mathrm{~S}_{3}$ (top panel, green) and ICDD 03-065-2023 for $Z \mathrm{nln}_{2} \mathrm{~S}_{4}$ (bottom panel, black). The intensity of the blue (top panel) and red (bottom panel) pattern was estimated for nanoplates with a diameter of $30 \mathrm{~nm}$ and thickness of $2.4 \mathrm{~nm}$, with a growth direction perpendicular to the $\langle 111\rangle$ direction of $\ln _{2} S_{3}$ and $\langle 001\rangle$ direction of $Z n n_{2} S_{4}$, respectively.

blue bars in Fig. 2. Both patterns are in good agreement with the experimental data. Only in the region of about $20^{\circ} 2 \theta$, we see a discrepancy between the calculated and the measured pattern. We observe in all samples a broad signal centered around $20^{\circ} 2 \theta$ with varying intensity (depending, e.g., on the number of cleaning steps). We do not find reflections in this region in the reference pattern of $\beta-\operatorname{In}_{2} S_{3}$. Furthermore, based on our estimated intensity values, we do not expect a strong reflection stemming from $\mathrm{ZnIn}_{2} \mathrm{~S}_{4}$ here, because it would correspond to (006) lattice planes, which are perpendicular to the short axis of the nanoplates. Thus, we assign it to ligand molecules, either bound to the surface of the nanoplates, or present as impurities in the samples. The lattice spacing for this peak calculated with the Bragg equation is $0.43 \mathrm{~nm}$, which corresponds to the closest possible distance between two carbon chains. Usually, the occurrence of this kind of signal indicates the presence of excess organic molecules, which were not sufficiently removed during the cleaning procedure. ${ }^{28}$ However, in the case of the nanoplates, we expect ligands on their surface to form a locally ordered layer. Therefore, we assign the reflection at $20^{\circ}$, at least partly, to the presence of the highly ordered ligand shell on the nanoplate's surface.

Taken together, the comparison of the measured and calculated intensity of the diffraction patterns confirms our assignment of the preferential growth direction of the nanoplates to be the $\langle 001\rangle$ and $\langle 111\rangle$ direction for the hexagonal $\mathrm{ZnIn}_{2} \mathrm{~S}_{4}$ and for the cubic $\beta-\mathrm{In}_{2} \mathrm{~S}_{3}$, respectively. However, the pronounced broadening of most of the reflections, results in relatively similar diffraction patterns for nanoplates with hexagonal $\mathrm{ZnIn}_{2} \mathrm{~S}_{4}$ and cubic $\beta$ - $\operatorname{In}_{2} \mathrm{~S}_{3}$ structure. Therefore it is not possible to unambiguously assign the crystallographic structure of the nanoplates with varying $\mathrm{Zn}$ content, based only on the XRD data.

Additional information about the internal structure of the $\mathrm{Zn}_{x} \mathrm{In}_{2} \mathrm{~S}_{3+x}$ (ZIS) nanoplates grown at different $\mathrm{Zn}$ concentrations in the parental solution can be gained from their lattice vibration spectra obtained by means of Raman spectroscopy. Raman spectra excited with $\lambda_{\text {exc }}=325.0 \mathrm{~nm}$ and $514.7 \mathrm{~nm}$ are shown in Fig. 3. Based on the UV-vis spectra (see below in the section "Optical properties"), the higher energy excitation is resonant for the samples containing Zn (Fig. 3a), while 514.7 $\mathrm{nm}$ provides resonance condition for exciting the vibrational Raman spectrum of the sample without $\mathrm{Zn}$, i.e., $\operatorname{In}_{x} \mathrm{~S}_{y}$ (Fig. $3 \mathrm{~b}$ ). Under $325 \mathrm{~nm}$ excitation, a broad scattering feature is observed in the spectrum with several distinct peaks, which increases in intensity as the amount of $\mathrm{ZnAc}_{2}$ in the reaction solution increases from $0.05 \mathrm{~g}$ up to $0.25 \mathrm{~g}$ (Fig. 3a). The spectrum of the nanoplates synthesized at the highest nominal $\mathrm{Zn}$ content $(0.25$ g) correlates well with the Raman spectrum of bulk ${ }^{29}$ and microcrystalline ${ }^{22} \mathrm{ZnIn}_{2} \mathrm{~S}_{4}$, reported previously. The most pronounced Raman band in Zn-containing samples peaks near $345 \mathrm{~cm}^{-1}$ under $\lambda_{\text {exc }}=325 \mathrm{~nm}$ and is close in frequency to the $\mathrm{F}_{\mathrm{u} 1}(\mathrm{LO})$ mode of the $\mathrm{ZnIn}_{2} \mathrm{~S}_{4}$ lattice. ${ }^{29}$ According to theory, this mode should not be Raman active, but its observation in bulk crystals has been explained as a breaking of selection rules due to defects. ${ }^{29}$ In our case, the internal non-stoichiometry of the nanoplates and especially their large surface-to-volume ratio might be the reason for activating forbidden Raman mode(s). Moreover, we observed an important role of defects and intragap states in our ZIS nanoplates (see the discussion in section "Optical properties"). The high surface-to-volume ratio of our nanoplates accounts for the observed broadening of the phonon bands. ${ }^{30,31}$ According to previous observations for other semiconductor NPs, the surface-induced broadening alone can make up as much as $30-50 \%$ of the Raman peak width. ${ }^{32}$ Additionally, the effect of phonon confinement is known to cause broadening and a shift of the optical phonon peaks (usually to lower frequencies as compared to bulk resonance) for semiconductor NPs smaller than $10 \mathrm{~nm}$ such as ours. ${ }^{33-35}$ According to other works on $\mathrm{ZnIn}_{2} \mathrm{~S}_{4},{ }^{36,37}$ Raman modes at 254 and $345 \mathrm{~cm}^{-1}$ stem mainly from vibrations of $\mathrm{S}$ atoms. An alternative origin of the $345 \mathrm{~cm}^{-1}$ Raman mode in the spectra of our $\mathrm{ZnIn}_{2} \mathrm{~S}_{4}$ nanoplates could be nanosized $\mathrm{ZnS}$ inclusions, because the main resonant Raman peak of small ZnS crystallites 
occurs in the range of $345-350 \mathrm{~cm}^{-1}$ and is due to longitudinal optical (LO) vibrations..$^{38}$ However, at the resonant excitation of II-VI nanocrystals ( $\lambda_{\text {exc }}=325 \mathrm{~nm}$ for $\mathrm{ZnS}$ ), also a second order peak (2LO) at approximately double the frequency is usually observed, ${ }^{38}$ even in alloys ${ }^{39}$ and ultrasmall crystallites $(<2 \mathrm{~nm}){ }^{40}$ The latter proves to be an efficient way of determining $\mathrm{ZnS}$ precipitations in multicomponent crystals, for example $\mathrm{Cu}_{2}-$ $\mathrm{ZnSnS}_{4}{ }^{38}$ For the $\mathrm{ZnIn}_{2} \mathrm{~S}_{4}$ nanoplates studied here no feature is observed near $700 \mathrm{~cm}^{-1}$ (Fig. 3a) indicating the absence of $\mathrm{ZnS}$ nano-inclusions. Moreover, no ZnS NPs were detected in these samples by TEM.

The most efficient resonant excitation is the near-bandgap one, which explains the absence of any Raman features for the sample without $\mathrm{Zn}$ at $\lambda_{\text {exc }}=325 \mathrm{~nm}$ (Fig. 3a) and the strong Raman scattering intensity at $\lambda_{\text {exc }}=514.7 \mathrm{~nm}$ (Fig. 3b). It contains an intense and relatively broad band at $300 \mathrm{~cm}^{-1}$ and weaker and narrower ones at 110,130,175 and $215 \mathrm{~cm}^{-1}$. These features do not match the strongest Raman bands of $\operatorname{In}_{2} \mathrm{~S}_{3}(246$ and $\left.365 \mathrm{~cm}^{-1}\right)^{41}$ or InS $\left(150 \mathrm{~cm}^{-1}\right) \cdot{ }^{42}$ The main Raman-active vibration of the most stable relevant oxide, $\operatorname{In}_{2} \mathrm{O}_{3}$, occurs at about $290 \mathrm{~cm}^{-1}$, ${ }^{41}$ but it cannot explain the rather strong and
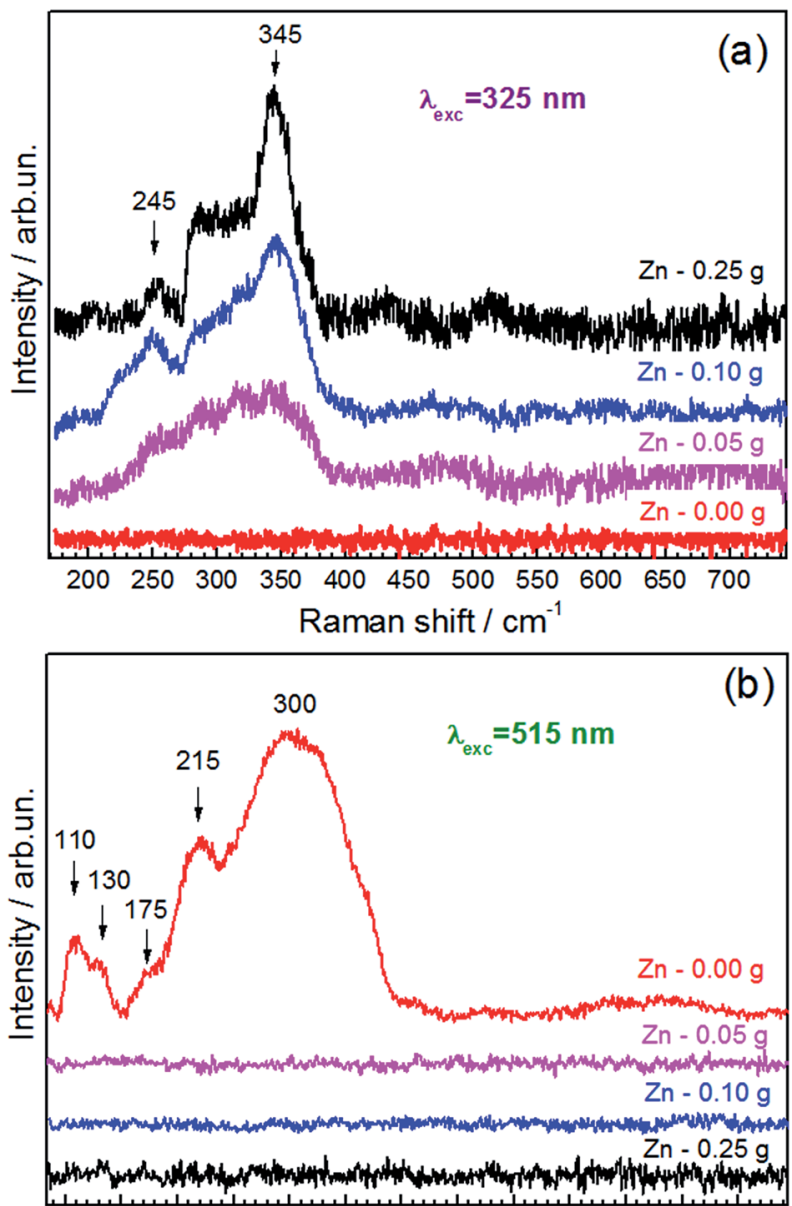

100150200250300350400450500550600650700 Raman shift $/ \mathrm{cm}^{-1}$

Fig. 3 Raman spectra of the nanoplates samples with different $\mathrm{Zn}$ concentration, recorded using $\lambda_{\text {exc }}=325 \mathrm{~nm}$ (a) and $514.7 \mathrm{~nm}$ (b). The features discussed in the text are marked by arrows. extremely broad structured feature observed for our $\operatorname{In}_{x} \mathrm{~S}_{y}$ nanoplates (Fig. 3b). The latter obviously results from an overlap of several vibrational modes. Based on the atomic ratio of In : $\mathrm{S}$ being near $2: 3$ (Table 1) and by taking into account the thickness of our nanoplates of only $2.5 \mathrm{~nm}$, the spectrum of our $\operatorname{In}_{x} \mathrm{~S}_{y}$ nanoplates can be assigned to the $\operatorname{In}_{2} \mathrm{~S}_{3}$ features broadened and shifted by the phonon confinement and surface effect, as explained above.

Fig. 4a shows an HRTEM image of a nanoplate. The corresponding fast Fourier transform (FFT) pattern (Fig. 4b) indicates that the entire nanoplate is mono-crystalline and grows in the $a b$ plane of the hexagonal $\mathrm{ZnIn}_{2} \mathrm{~S}_{4}$ structure. Our findings are in good agreement with the XRD data, exhibiting a pronounced and narrow (110) reflection with missing reflections corresponding to (002) and (004) planes of $\mathrm{ZnIn}_{2} \mathrm{~S}_{4}$ (Fig. 2). This is exactly what we expect for thin nanoplates growing in the $a b$ plane. While the lattice planes can hardly be seen in isolated nanoplates because of their low thickness, moiré fringes are observed when nanoplates overlap, which further confirms the mono-crystalline nature of the nanoplates. Fig. 4c shows such a moiré pattern with hexagonal symmetry, resulting from two overlapping nanoplates rotated by $2^{\circ}$ with respect to each other. In the FFT of this region (Fig. $4 \mathrm{~d}$ ), the patterns confirm a $2^{\circ}$ rotation of the nanoplates. A hexagonal pattern in the center of the FFT reflects the symmetry of the moire fringes and their spacing of $5 \mathrm{~nm}$.

\section{Optical properties}

A careful investigation of the effect of composition on the optical properties of three samples of ZIS nanoplates with increasing Zn content was performed through multiple optical spectroscopic measurements, such as continuous wave $(\mathrm{CW})$ absorption and

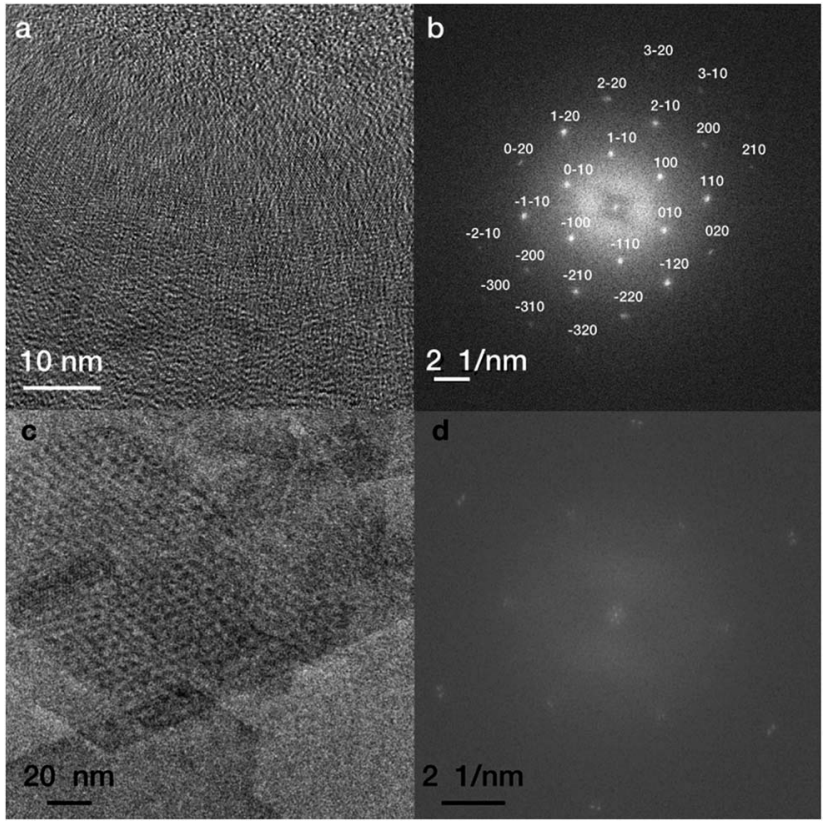

Fig. 4 HRTEM image of a single nanoplate (a) and the corresponding FFT (b), which can be indexed to the hexagonal $Z n n_{2} S_{4}$ viewed along the $\langle 001\rangle$ zone axis. TEM image of overlaying nanoplates exhibiting a moiré pattern (c), and an FFT of this region (d). 
photoluminescence (PL) as well as time resolved (TR) PL measurements. In the $\mathrm{CW}$ absorption analysis we observe a steep absorption onset around $3 \mathrm{eV}(413 \mathrm{~nm})$ for all samples with a weak absorption extending to the red (Fig. 5). The Tauc's plot, ${ }^{43}$ i.e., absorbance squared versus energy, gives a linear relationship, which corresponds to a direct transition (see ESI Fig. S2 $\dagger$ ). The extrapolation of the linear trend to the energy axis determines the band gap. Due to a large contribution from scattering, a precise analysis of band gap shifts with changing composition is not possible. Nevertheless, a direct transition at around $3 \mathrm{eV}$ can be extracted in all samples, relatively close to the bulk bandgap of $\mathrm{ZnIn}_{2} \mathrm{~S}_{4}$ at $2.8 \mathrm{eV} \cdot{ }^{\mathbf{4 4}}$ In line with the recent publication by Chen et al. ${ }^{\mathbf{4 5}}$ we expect a composition dependent bandgap, i.e., a blue shift with increasing incorporation of zinc

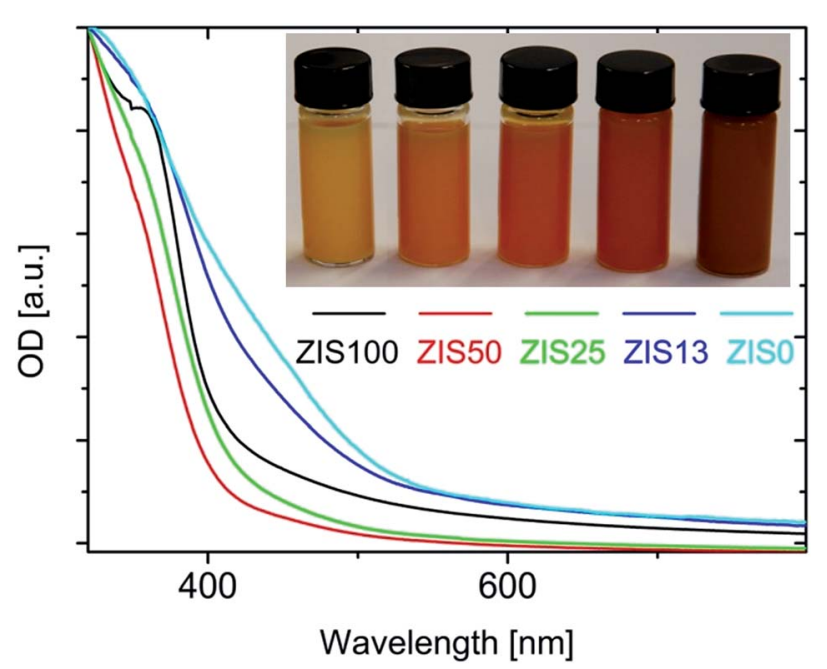

Fig. 5 Normalized absorption spectra of nanoplates synthesized with different amount of $\mathrm{ZnAc}_{2}$ in the reaction solution (the kink in the black curve about $350 \mathrm{~nm}$ is an instrumental artifact). The inset shows a photograph of the corresponding solutions. over indium. Notably, a significant contribution to the absorption is observed below gap, which is largely due to scattering of the samples, however, a significant contribution might also correspond to defect states causing an Urbach tail extending into the forbidden gap. ${ }^{46}$ Indeed, in past investigations on $\mathrm{ZnIn}_{2} \mathrm{~S}_{4}$ films, a density-of-state tail below the conduction band (CB) reaching into the forbidden gap was observed. ${ }^{\mathbf{4 6}}$

To better understand this we performed CW PL measurements on the samples by exciting at $3.1 \mathrm{eV}$ (400 nm - above band gap, blue curves in Fig. 6) and at $2.8 \mathrm{eV}$ (450 $\mathrm{nm}$ - below band gap, red curves in Fig. 6). The resulting PL shows a relatively broad peak with a large Stokes shift. A blue shift of the PL as well as an increase in intensity with increasing content of $\mathrm{Zn}$ was found for both values of the excitation wavelength. This is visualized by the images of the sample cuvettes illuminated with a handheld UV-lamp (Fig. 6, inset). A careful analysis of the PL spectra allowed a fitting with one or two Gaussians. A common peak at around $2.1 \mathrm{eV}(590 \mathrm{~nm})$ in all $\mathrm{Zn}$ containing samples was found with rather low intensity that is increasing with the $\mathrm{Zn}$ content. For the two samples with the highest $\mathrm{Zn}$ content, an additional intense peak arises at $2.3 \mathrm{eV}(540 \mathrm{~nm})$ and $2.5 \mathrm{eV}(496 \mathrm{~nm})$, respectively. The excitation below band gap with $2.8 \mathrm{eV}(450 \mathrm{~nm})$ shows a similar trend, however, with a much lower intensity (factor of approximately five): a Gaussian peak at around $2.1 \mathrm{eV}$ in each sample with low intensity and an additional peak for the two higher samples with the highest $\mathrm{Zn}$ content peaking at around $2.3 \mathrm{eV}$ and $2.41 \mathrm{eV}$, respectively.

The large Stokes shift observed is indicative of radiative carrier recombination from intra-gap levels, or donor-acceptor pair recombination, and not from the transition between quantized energy levels in conduction and valence bands. ${ }^{47}$ Indeed, we do not observe any PL corresponding to the inter-gap recombination (at around $3 \mathrm{eV}$ ). Notably, the excitation with 450 $\mathrm{nm}$, which is below the bandgap, results in similar PL behavior. The latter indicates the presence of absorption and subsequent

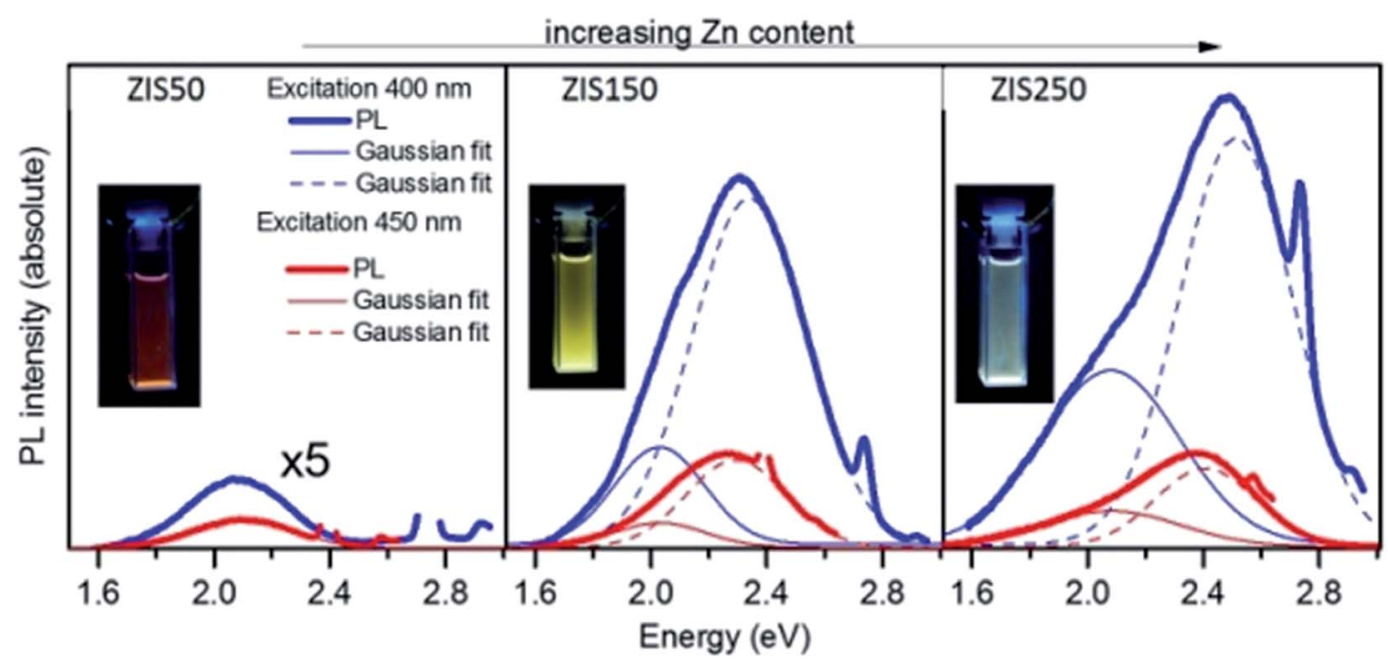

Fig. 6 PL spectra for three samples with increasing Zn content (increasing from ZIS50, ZIS150, to ZIS250). Blue curves correspond to excitation at $400 \mathrm{~nm}$, while red curves correspond to excitation at $450 \mathrm{~nm}$. The thick solid curves represent the experimental data, and the thin solid and dotted curves the Gaussian fits. 
emission from states below the band gap. This is in agreement with the Urbach tail observed in the absorption spectra.

The results of the PL measurements are interpreted in the following way: the electron excited from the valence band or from the acceptor levels to the conduction band (CB) thermalizes to and below the bottom of the CB. The electron then recombines with the hole, trapped at an acceptor. Due to the high defect density of ternary compounds based on $\mathrm{Zn}$, In, and S, a manifold of intrinsic defects might be responsible for such donor and acceptor levels, e.g., $\mathrm{Zn}$ ions in the In sublattice, In ions in the $\mathrm{Zn}$ sublattice, and vacant sites in both $\mathrm{Zn}$ and In sublattices. These defects characterize the electronic properties of the material and give rise to a manifold of additional electronic levels. As such, numerous defect levels inside the forbidden energy gap are expected. ${ }^{44}$ Interestingly, in our work, we observe not only below gap emission, but two distinct contributions peaking at 2.1 and around 2.3-2.5 eV. Similar results were obtained by A. Serpi, ${ }^{\mathbf{4}}$ who discusses the existence of a manifold of impurity levels in films of $\mathrm{ZnIn}_{2} \mathrm{~S}_{4}$, as determined via photoconductivity measurements. Aside from the band gap peak, three other peaks at around $1.4 \mathrm{eV}$, $2.35 \mathrm{eV}$, and $2.55 \mathrm{eV}$ were observed in the photoconductivity experiments. These reflect three impurity levels in the forbidden energy gap taking part in the photoconductivity process. ${ }^{\mathbf{4 4}}$

Resolving PL emission temporally as well as spectrally can often yield further insight into the electronic structure and behavior of a material system. As such, TR PL measurements were performed at all four samples (ZIS250, ZIS150, ZIS50 and Zn-free ZIS0) with excitation at $3.1 \mathrm{eV}$ (400 nm). In contrast to the CW PL, all samples - including the Zn-free ZIS0 - were seen to have some emission in TR measurements. This discrepancy can most likely be attributed to the higher excitation fluences and detection efficiency of the TR PL experimental setup with respect to the CW PL setup.

Inspection of the TR PL dynamics showed that they could be well described by either mono- (in the case of ZISO) or biexponential decays with short and long decay constants of the order of $1 \mathrm{~ns}$ and $100 \mathrm{~ns}$, respectively. These two emission components were, however, strongly spectrally overlapped, precluding further individual analysis based on spectral selection. As such, a global analysis approach was taken, with the entire TR PL map being fitted using the biexponential function,

$$
\mathrm{PL}(\varepsilon, t)=\sum_{i=1}^{n} A_{i}(\varepsilon) \mathrm{e}^{-t / \tau_{i}}, n=2
$$

with the amplitudes at each energy, $A_{i}(\varepsilon)$, and the decay constants, $\tau_{i}$, as fit parameters. The resulting fits showed a low sum of squares error values and evenly distributed residuals (see ESI Fig. S3†), confirming the high fit quality and the inherently assumed energy independence of the decay constants $\left(\tau_{i} \neq \tau_{i}(\varepsilon)\right)$. The amplitude spectra, $A_{i}(\varepsilon)$ are presented in Fig. 7, and clearly show the spectral deconvolution of the short $\left(\tau_{1}\right)$ and long $\left(\tau_{2}\right)$ emission components. It should be noted that despite low amplitudes, the longer lifetime of the $\tau_{2}$ component typically resulted in it being the dominant emission observed in CW measurements (see ESI Fig. S4†).

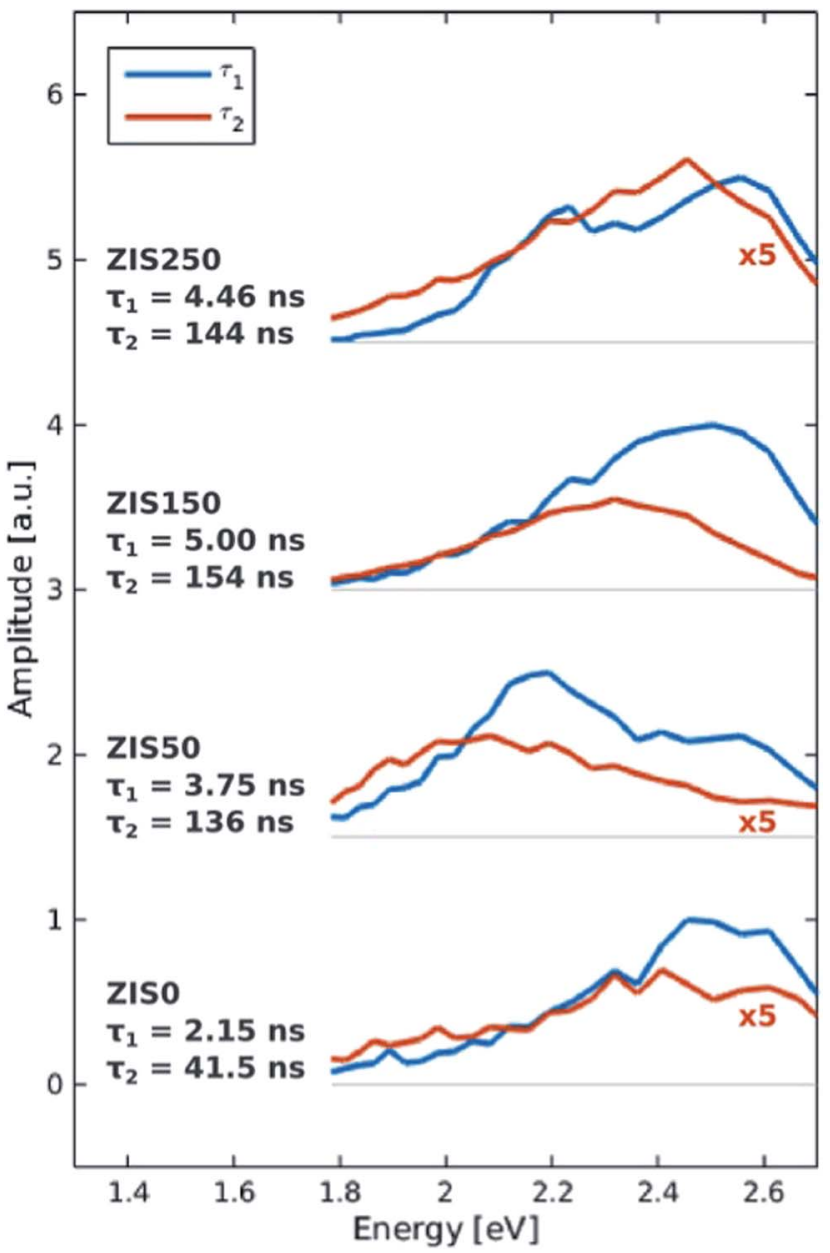

Fig. 7 TR PL amplitude spectra. Each spectrum, $A_{i}(\varepsilon)$, is associated with a mono-exponential decay of time constant $\tau_{i}$, such that the TR PL signal, $\operatorname{PL}(\varepsilon, t)$, is given by the sum of the spectrum-decay product. Spectra have been scaled and offset for clarity.

Whilst the Zn-free sample (ZIS0) shows a weak $\tau_{2}$ component with almost identical spectral shape to the $\tau_{1}$ component, the incorporation of $\mathrm{Zn}$ can be seen to increase the amplitude of the $\tau_{2}$ spectrum and change its shape. This shows that the short component is intrinsic intra-gap ZIS emission, whilst the long emission component can be associated to states arising from the presence of $\mathrm{Zn}$. Given their extremely low energy ( $c f$., bandgap $\sim 3 \mathrm{eV}$ ) and long lifetime, such states are likely to be deep intra-gap trap states arising from defect sites due the incorporation of $\mathrm{Zn}$ into the crystal lattice.

The peak of the $\tau_{2}$ spectrum can be seen to blue-shift with increasing $\mathrm{Zn}$ content, from circa $1.98 \mathrm{eV}$ (625 nm) in ZIS50 to $2.43 \mathrm{eV}(510 \mathrm{~nm})$ in ZIS250. This is consistent with the blue-shift seen in CW PL, and indicates a reduced trap depth. Furthermore, the lifetime of both short and long emission components can be seen to increase marginally, suggesting a reduction in non-radiative decay channels. This can also be observed in the CW PL measurements (Fig. 6) as a significant increase in the PL quantum yield with increasing $\mathrm{Zn}$ content. Taken together, all these factors suggest that the increased incorporation of $\mathrm{Zn}$ into the lattice results in an improvement in crystal quality. 
In summary, with increasing $\mathrm{Zn}$ incorporation, more intragap states are created. These participate not only in radiative sub-band gap recombination, but also simultaneously decrease non-radiative defect states that might, for example, originate from vacancies in the crystal structure.

\section{Photochemical behavior of zinc indium sulfide nanoplates}

The photochemical activity of ZIS nanoplates was evaluated by the degradation of rhodamine 6G (R6G) under UV light. R6G is a non-biodegradable and possibly carcinogenic ${ }^{48}$ organic dye of a remarkably high photostability, used, e.g., as gain medium in dye lasers or in paper, textile, or printing industry. Furthermore, it is a good model system for many other organic pollutants.

ZIS was demonstrated previously to be a suitable photocatalyst for the degradation of organic dyes, among others R6G,$^{\mathbf{1 3}, 15}$ therefore, we also used this reaction to evaluate the photochemical activity of our nanoplates. In Fig. 8, absorption spectra are shown, which were taken at different stages of the decomposition process of R6G in the presence of ZIS nanoplates under UV irradiation. The spectrum of R6G is dominated by an intense resonance at around $530 \mathrm{~nm}$ that decreases in intensity with reaction time. A pure R6G solution without the nanoplates remains stable under the same conditions, and no decrease in the concentration of the dye can be observed. During the degradation process of R6G, the absorption maximum of the

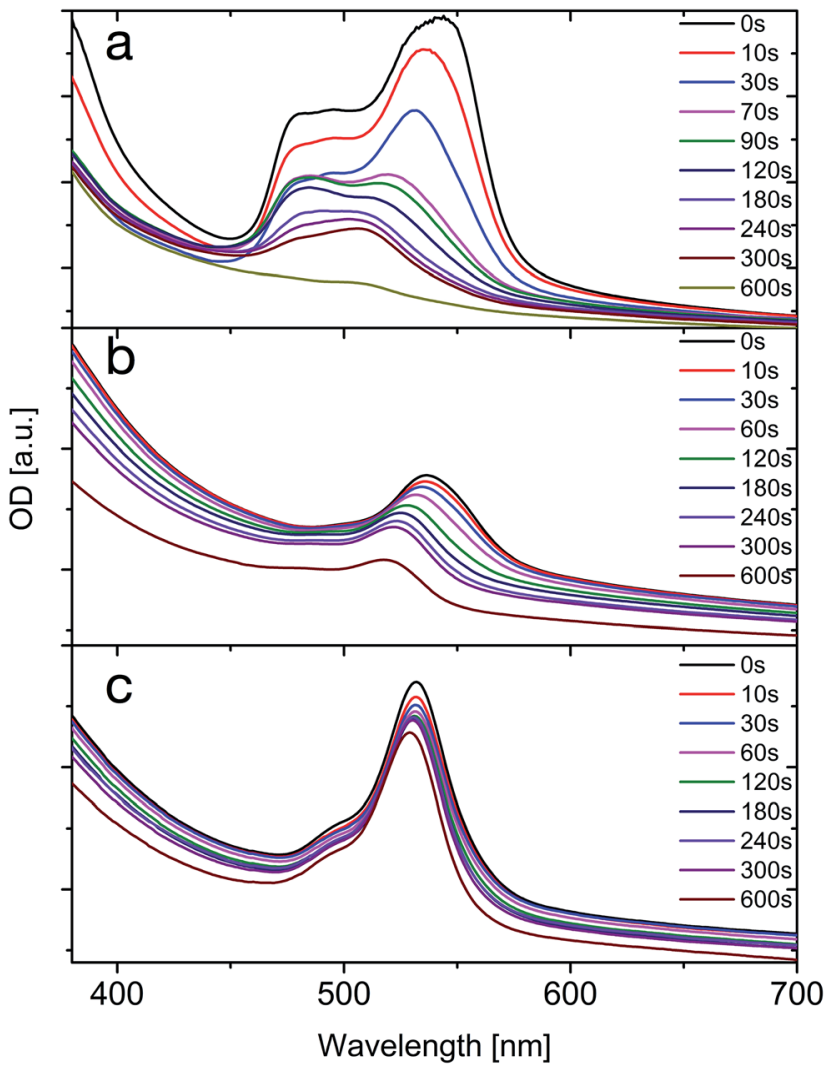

Fig. 8 Absorption spectra taken at different times during decomposition of R6G with samples ZIS50 (a), ZIS25 (b), and ZIS13 (c). dye shifts to shorter wavelengths, and an irradiation time of 10 min is sufficient to decompose the dye completely when Zn-rich nanoplates are used (Fig. 8a). The final absorption spectrum taken from this sample (600 s) does not exhibit any features typical of R6G. Thus, the degradation of R6G in the presence of our ZIS nanoplates is considerably faster as compared to results reported in literature, where photocatalytic experiments with ZIS microspheres were conducted on a time scale of several hours. ${ }^{16-19}$ We attribute this to the high specific surface area of ultrathin nanoplates used in our study.

The decomposition of the dye is considerably slower when nanoplates with a smaller $\mathrm{Zn}$ content are used (Fig. $8 \mathrm{~b}$ and c). We exclude a smaller number of photons available for photoexcitation to explain the observed behavior, as the absorption of $\mathrm{Zn}$-rich nanoplates is blue shifted with respect to particles containing less zinc. Therefore, we conclude that the difference in the photochemical activity of the nanoplates results from different electronic or surface properties, depending on the composition of the particles. The formation of intra-gap states in the structure may also play a role here. As we have seen from our CW and TR PL results, the contribution of long lived intra-gap states increases with increasing amounts of $\mathrm{Zn}$ in the structure, simultaneously leading to an improvement in crystal quality, i.e., a removal of non-radiative defect states. A careful evaluation of the role of $\mathrm{Zn}$ incorporation, intra-gap formation, and defect states in the structure and, in particular, on the photocatalytic activity is ongoing. Furthermore, a correlation of the high surface-to-volume ratio on both should deliver a better understanding of the photocatalytic processes responsible for the highly increased photocatalytic activity of our ultrathin ZIS nanoplates.

\section{Conclusions}

We developed a synthesis for ultrathin, colloidal, single crystalline $\mathrm{Zn}_{x} \mathrm{In}_{2} \mathrm{~S}_{3+x}$ nanoplates with varying $\mathrm{Zn}$ content between $x=0$ and $x=1$. The structure of the $\mathrm{Zn}$-free particles was assigned to $\beta$ - $\operatorname{In}_{2} S_{3}$ based on the powder X-ray diffraction and Raman spectroscopy data. Particles containing $\mathrm{Zn}$ exhibit the hexagonal $\mathrm{ZnIn}_{2} \mathrm{~S}_{4}$ structure. The formation of the nanoplates was facilitated by the presence of copper ions in the reaction solution, and their role in the growth process and the growth mechanism will be evaluated in further studies. The optical and photochemical properties of the nanoplates depend on their $\mathrm{Zn}$ content. A generally much higher photocatalytic activity for the degradation of rhodamine $6 \mathrm{G}$ was found for our ultrathin $\mathrm{Zn}_{x} \mathrm{In}_{2} \mathrm{~S}_{3+x}$ nanoplates as compared to thicker $\mathrm{Zn}_{x^{-}}$ $\mathrm{In}_{2} \mathrm{~S}_{3+x}$ nanostructures. This might result from the unique shape of our nanoplates, which have a thickness of only 2.5 $\mathrm{nm}$ and a diameter of up to $100 \mathrm{~nm}$, providing a high surfaceto-volume ratio. Degradation was also seen to improve with increasing $\mathrm{Zn}$ content. We speculate that the formation of intra-gap states with increasing $\mathrm{Zn}$ content and the simultaneous decrease in non-radiative defect states might play a major role on the photocatalytic activity and deserves a further in depth evaluation. 


\section{Acknowledgements}

We gratefully acknowledge funding of the EWE Research Group "Thin Film Photovoltaics" by the EWE AG, Oldenburg. The authors acknowledge also the financial support by the MWN (Material World Network) Program between the National Science Foundation of the U.S. and the Deutsche Forschungsgemeinschaft (Grant No. Li 580/8-1).

\section{Notes and references}

1 A. R. Tao, S. Habas and P. Yang, Small, 2008, 4, 310-325.

2 C. Burda, X. Chen, R. Narayanan and M. a. El-Sayed, Chem. Rev., 2005, 105, 1025-1102.

3 C. B. Murray, C. R. Kagan and M. G. Bawendi, Annu. Rev. Mater. Sci., 2000, 30, 545-610.

4 P. Alivisatos, J. Phys. Chem., 1996, 100, 13226-13239.

5 S. Kumar and T. Nann, Small, 2006, 2, 316-329.

6 J. Park, J. Joo, S. G. G. Kwon, Y. Jang and T. Hyeon, Angew. Chem., Int. Ed., 2007, 46, 4630-4660.

7 F.-J. Fan, L. Wu and S.-H. Yu, Energy Environ. Sci., 2014, 7, 190.

8 D. Aldakov, A. Lefrançois and P. Reiss, J. Mater. Chem. C, 2013, 1, 3756-3776.

9 J. Kolny-Olesiak, CrystEngComm, 2014, 16, 9381-9390.

$10 \mathrm{~J}$. Kolny-Olesiak and H. Weller, ACS Appl. Mater. Interfaces, 2013, 5, 12221-12237.

11 E. Witt and J. Kolny-Olesiak, Chem.-Eur. J., 2013, 19, 97469753.

12 Y. Zhao and C. Burda, Energy Environ. Sci., 2012, 5, 55645576.

13 Y. Chen, S. Hu, W. Liu, X. Chen, L. Wu, X. Wang, P. Liu and Z. Li, Dalton Trans., 2011, 40, 2607-2613.

14 W. Seo, R. Otsuka, H. Okuno, M. Ohta and K. Koumoto, J. Mater. Res., 1999, 14, 4176-4181.

15 Y. Chen, R. Huang, D. Chen, Y. Wang, W. Liu, X. Li and Z. Li, ACS Appl. Mater. Interfaces, 2012, 4, 2273-2279.

16 S. Shen, L. Zhao, Z. Zhou and L. Guo, J. Phys. Chem. C, 2008, 112, 16148-16155.

17 B. Chai, T. Peng and P. Zeng, J. Phys. Chem. C, 2011, 115, 6149-6155.

18 Z. Mei, S. Ouyang, D.-M. D. Tang, T. Kako, D. Golberg and J. Ye, Dalton Trans., 2013, 42, 2687-2690.

19 S. Peng, L. Li, Y. Wu, L. Jia, L. L. Tian, M. Srinivasan, S. Ramakrishna, Q. Yan and S. G. Mhaisalkar, CrystEngComm, 2013, 15, 1922.

20 L. Shi, P. Yin and Y. Dai, Langmuir, 2013, 29, 12818-12822.

21 X. Gou, F. Cheng, Y. Shi, L. Zhang, S. Peng, J. Chen and P. Shen, J. Am. Chem. Soc., 2006, 128, 7222-7229.

22 S. Shen, L. Zhao and L. Guo, Int. J. Hydrogen Energy, 2010, 35, 10148-10154.

23 M. Kruszynska, J. Parisi and J. Kolny-Olesiak, Z. Naturforsch., A: Phys. Sci., 2014, 69, 446-450.

24 M. Kruszynska, H. Borchert, J. Parisi and J. Kolny-Olesiak, J. Am. Chem. Soc., 2010, 132, 15976-15986.
25 M. Kruszynska, H. Borchert, A. Bachmatiuk, M. H. Rümmeli, B. Buechner, J. Parisi and J. Kolny-Olesiak, ACS Nano, 2012, 6, 5889-5896.

26 M. Kruszynska, H. Borchert, J. Parisi and J. Kolny-Olesiak, J. Nanopart. Res., 2011, 13, 5815-5824.

27 J. Li, M. Bloemen, J. Parisi and J. Kolny-Olesiak, ACS Appl. Mater. Interfaces, 2014, 6, 20535-20543.

28 J. Tang, G. Konstantatos, S. Hinds, S. Myrskog, A. G. Pattantyus-abraham, J. Clifford and E. H. Sargent, ACS Nano, 2009, 3, 331-338.

29 G. Baldini, R. L. Aggarwal, B. Lax, S. H. Shin and J. C. Tsang, Lett. Nuovo Cimento Soc. Ital. Fis., 1972, 5, 1062-1066.

30 M. Dimitrievska, A. Fairbrother, E. Saucedo, A. PérezRodríguez and V. Izquierdo-Roca, Appl. Phys. Lett., 2015, 106, 073903.

31 V. Dzhagan, I. Lokteva, C. Himcinschi, X. Jin, J. KolnyOlesiak and D. R. T. Zahn, Nanoscale Res. Lett., 2011, 6, 79.

32 V. M. Dzhagan, M. Y. Valakh, O. E. Raevska, O. L. Stroyuk, S. Y. Kuchmiy and D. R. T. Zahn, Nanotechnology, 2009, 20, 365704 .

33 C. Camus, E. Rudigier, D. Abou-Ras, N. a. Allsop, T. Unold, Y. Tomm, S. Schorr, S. E. Gledhill, T. Köhler, J. Klaer, M. C. Lux-Steiner and C.-H. Fischer, Appl. Phys. Lett., 2008, 92, 101922.

34 D. O. Sigle, J. T. Hugall, S. Ithurria, B. Dubertret and J. J. Baumberg, Phys. Rev. Lett., 2014, 113, 087402.

35 A. C. A. Silva, E. S. F. Neto, S. W. da Silva, P. C. Morais and N. O. Dantas, J. Phys. Chem. C, 2013, 117, 1904-1914.

36 B. Fontal, S. A. Lopez-Rivera, L. Martınez and W. Giriat, Semicond. Sci. Technol., 1996, 11, 1056-1058.

37 S. A. Lopez-Rivera, L. Martinez, B. Fontal, W. Giriat and F. Medina, Semicond. Sci. Technol., 1995, 10, 645-652.

38 A. Fairbrother, V. Izquierdo-Roca, X. Fontané, M. Ibáñez, A. Cabot, E. Saucedo and A. Pérez-Rodríguez, CrystEngComm, 2014, 16, 4120.

39 A. V. Kozytskiy, O. L. Stroyuk, S. Y. Kuchmiy, V. M. Dzhagan, D. R. T. Zahn, M. a. Skoryk and V. O. Moskalyuk, J. Mater. Sci., 2013, 48, 7764-7773.

40 V. M. Dzhagan, M. Y. Valakh, C. Himcinschi, A. G. Milekhin, D. Solonenko, N. a. Yeryukov, O. E. Raevskaya, O. L. Stroyuk and D. R. T. Zahn, J. Phys. Chem. C, 2014, 118, 19492-19497.

41 A. Datta, S. K. Panda, D. Ganguli, P. Mishra and S. Chaudhuri, Cryst. Growth Des., 2007, 7, 163-169.

42 N. M. Gasanly, H. Özkan, A. Aydinli and I. Yilmaz, Solid State Commun., 1999, 110, 231-236.

43 J. I. Pankove, Optical Processes in Semiconductors, Courier Corporation, 2012.

44 A. Serpi, J. Phys. D: Appl. Phys., 1976, 9, 1881.

45 B. Chen, S. Chang, D. Li, L. Chen, Y. Wang, T. Chen, B. Zou, H. Zhong and A. L. Rogach, Chem. Mater., 2015, 27, 59495956.

46 V. F. Zhitar, V. Y. Raylyan and S. I. Radautsan, Nuovo Cimento Soc. Ital. Fis., D, 1983, 2, 1919-1922.

47 T. Ogawa, T. Kuzuya, Y. Hamanaka and K. Sumiyama, J. Mater. Chem., 2010, 20, 2226.

48 Hazardous Substances Data Bank - Rhodamin 6G. 